\title{
Investigation of divertor movement during disruptions in ASDEX Upgrade
}

\author{
M. Dibona, I. Zammuto ${ }^{a}$ A. Herrmanna, S. Vorbrugga ${ }^{a}$ ASDEX Upgrade Team \\ aMax-Planck-Institute for Plasma Physics, Boltzmannstr. 2, 85748 Garching, Germany
}

The divertor serves as the main power exhaust of tokamaks. Hence the target tiles in the divertor must be carefully aligned to prevent leading edges which would result in higher power deposition and subsequent melting. The outer strike line in the lower divertor of ASDEX Upgrade is located on the assembly 1, which consists of the target tiles, the cooling plates and the support structure. Since the transition to the tungsten optimized divertor design of the divertor in 2014, it has been observed that the assembly 1 and the underlying frame are displaced over the course of an experimental campaign. The attachment of the assembly has been modified several times to prevent this displacement. However, a complete suppression of the movement was not achieved. The reason for the displacement is suspected to be due to induced currents and the resulting jxB forces during disruptions. This was investigated using a full 3D transient model of the ASDEX Upgrade coil system and a model of the assembly 1 with frame and vacuum vessel. The different assembly modifications and several current quench times were simulated, resulting in forces of up to $5.5 \mathrm{kN}$ and torques up to $6.3 \mathrm{kNm}$. These forces were then used in a 3D transient structural model of the assembly to investigate the resulting displacements. It was found that displacements occur in all cases but they vary between $0.25 \mathrm{~mm}$ and 0.75 $\mathrm{mm}$.

Keywords: divertor, disruption, electromagnetic force

\section{INTRODUCTION}

The divertor is an important component of most fusion devices, as it serves as power exhaust for the machine. This is achieved by diverting the plasma outside the last closed flux surface into an outer and inner leg which are then guided onto special target tiles. The strike lines, on which the plasma hits the tiles, are characterized by heat fluxes of up to $15 \mathrm{MW} / \mathrm{m}^{2}$ in ASDEX Upgrade [1]. For this reason the tiles are either made of graphite [2] or tungsten [3] as these materials can withstand these heat fluxes. The alignment of the target tiles is also of great importance as leading edges are exposed to higher heat fluxes than the tile surface. This can lead to severe damage or destruction of entire tiles. For this reason, the tungsten divertor in ASDEX Upgrade (AUG) is carefully aligned after every maintenance break to prevent leading edges and to ensure proper shading across neighboring divertor assemblies. The divertor of AUG was originally equipped with graphite tiles. Since graphite is not suitable as target material for reactors, and thus for ITER, due to hydrogen co-deposition, AUG was stepwise transformed to a tungsten first wall [4]. The current design of the lower divertor (Div-III [5]), with solid tungsten tiles on the outer divertor, was introduced in 2014 to expand the experimental capabilities of AUG. Since this change it was observed that the divertor assembly 1 , which holds the target tiles for the outer strike line, was displaced in radial direction during an experimental campaign. Fig. 1 shows the radial offsets of the assemblies with respect to their neighboring assemblies in each toroidal sector directly before and directly after the experimental campaign between February and July 2017, which stands exemplary for the behavior in the campaigns prior. The green line represents the status of the assemblies after maintenance and the red line indicates the positions directly after opening the machine. It can be seen that during the experiments displacements between $0 \mathrm{~mm}$ and $0.35 \mathrm{~mm}$ occurred. Depending on the direction of the displacement, this increased or decreased shading
43 between the assemblies, resulting in leading edges,

44 higher heat fluxes and partial melting of tiles.

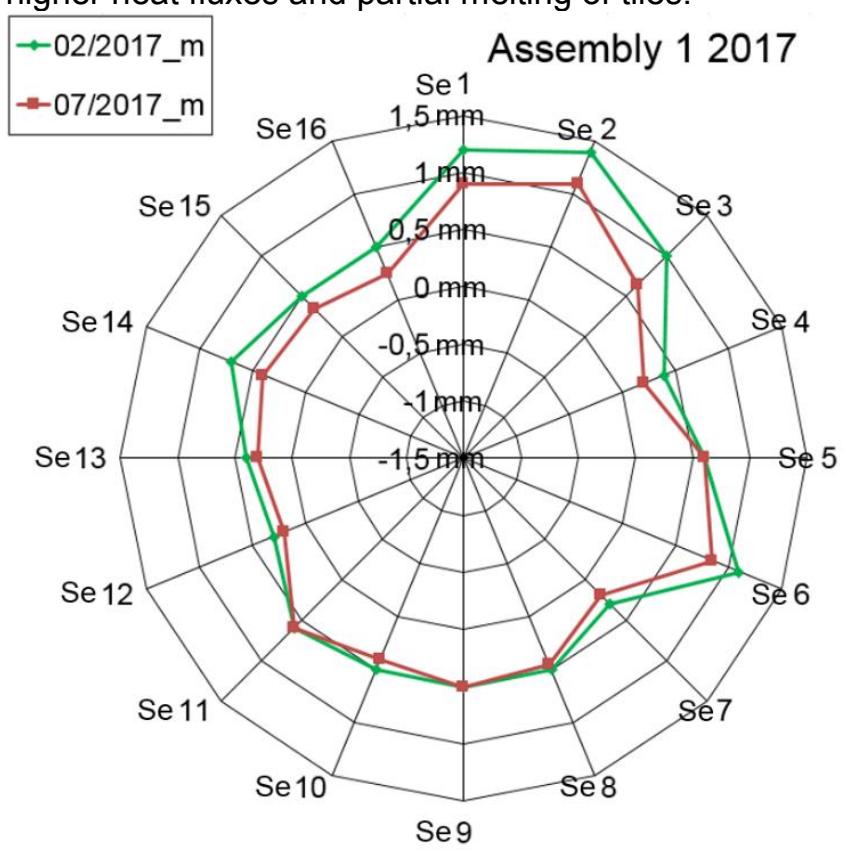

Figure 1: Radial shadowing of assemblies relative to their 47 neighboring ones before (green) and after (red) the 48 experimental campaign 02/2017 - 07/2017

49 As of 2017, the assembly 1 consists of a support 50 structure (Fig. 2 (1)) which holds two cooling plates (2). 51 A pipe (3) for the cooling water is attached to these 52 cooling plates. The cooling water is fed through a flange 53 (4a) into the pipe and exits the assembly through a 54 second flange (4b). Both flanges are mounted to the 55 support structure. Each assembly holds eight tungsten 56 tiles (5), four on each cooling plate. A $2 \mathrm{~mm}$ sheet of 57 Papyex (6) between the tiles and the cooling plate 58 serves as thermal conductor. The tiles and the Papyex 59 are firmly pulled against the cooling plates by clamps 60 above (7a) and below (7b) the tiles. The assembly 1 is 61 held in place by the two water flanges which are 62 attached to an underlying frame and by two sockets (left 63 (8b) and right (8a)) which are mounted directly to the 64 vacuum vessel. The frame itself is also attached to the 65 AUG vacuum vessel. Except the tiles and the Papyex, 
1 all components of the assembly 1 are made from

2 stainless steel, as is the supporting frame.

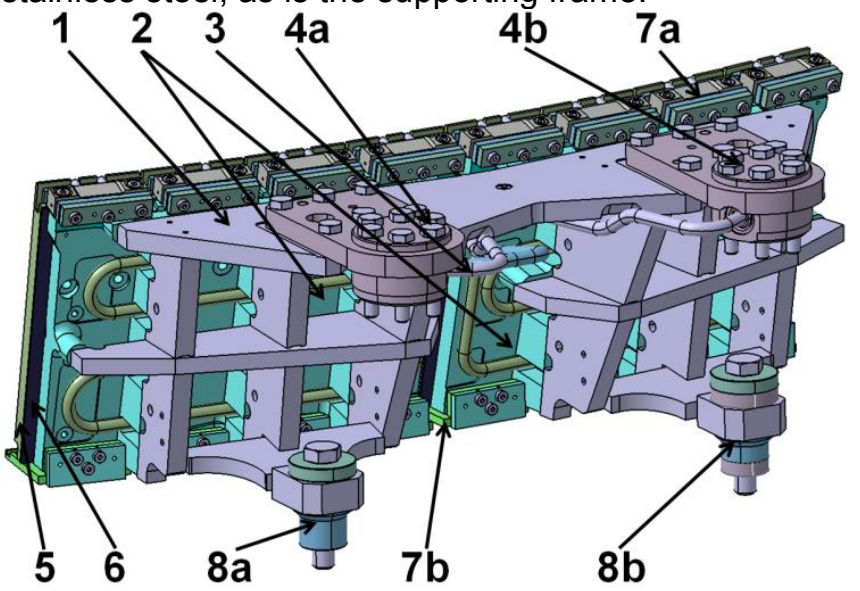

Figure 2: CAD model of 2017 assembly 1 with the support structure (1), cooling plates (2), cooling pipe (3), water flanges (4a, 4b), tungsten tiles (5), Papyex (6), clamps (7a, $7 b)$ and sockets $(8 a, 8 b)$

8 The sockets of the assembly 1 have undergone a series

9 of modifications due to installation space and to prevent

10 movement. These different versions, in which no socket,

11 one socket or both sockets are electrically insulated, are 12 represented in the simulated cases.

13 The reason for this movement is suspected to be jxB 14 forces resulting from induced currents during 15 disruptions. Other reasons for the displacement like 16 thermal stress or halo currents are not investigated since the temperature of the support structure is never elevated significantly and halo currents create a uniform force on the assembly, which would result an increased shading of one neighboring assembly and a decreased shading of the other one. The investigation of the eddy currents and their effect on the assembly are described in this paper. This includes the electromagnetic model to calculate the forces and torques on the assembly 1 and a finite element model to simulate the displacement of the assembly.

\section{ELECTROMAGNETIC MODEL}

This analysis was carried out in ANSYS Maxwell. A full $3 D$ model of the AUG coil system was used for this investigation, consisting of 16 toroidal field coils, 5 ohmic heating coils, 12 vertical field coils and 4 outer and 2 inner control coils [6]. The space between the single coils and between the coils and the divertor assembly was set to be vacuum. All boundaries were regarded as insulated. The material of the coils was set to copper. The plasma was represented by a perfectly conducting coil with one single winding. This filamentary model is an approximation, since the plasma itself experiences no force during the simulation. A different approach [7] using CarMaONL has shown good results. Typical static values were used for the currents in the coils except for the currents in the plasma $I_{P}(t)$ (Eq. 1) and the inner control coils $I_{C}(t)$ (Eq. 3). The current decay time constant of the plasma current $T_{P}$ (Eq. 2) was set to create a certain change of the poloidal magnetic field.

The initial plasma current $I_{P, 0}$ was set to $1.6 \mathrm{MA}$ and the plasma dimensions $a$ and $b$ were set to $0.5 \mathrm{~m}$ and 0.8 $\mathrm{m}$. The change rate of the poloidal magnetic field $\dot{B}_{p}$ was set to increase from $50 \mathrm{~T} / \mathrm{s}$ to $200 \mathrm{~T} / \mathrm{s}$ in steps of $25 \mathrm{~T} / \mathrm{s}$. The time constants were derived from the average current decay times for the single $B_{p}$ change rates. The relation between the poloidal magnetic field and the plasma current, using the mean plasma radius $\sqrt{a \cdot b}$, was used to determine the current decay times. Spacial variations of the plasma were neglected.

\begin{tabular}{|c|c|}
\hline$I_{P}(t)=I_{P, 0} \cdot e^{t / \tau_{P}}$ & (1) \\
\hline$\tau_{P}=\frac{I_{P, 0} \cdot \mu_{0}}{\ln \left(\frac{1 A}{I_{P, 0}}\right) \cdot \dot{B_{p}} \cdot 2 \pi \cdot \sqrt{a \cdot b}}$ & $(2)$ \\
\hline$\tau_{C}=\frac{I_{C}(t)=1 A \cdot e^{t / \tau_{C}}}{\ln \left(\frac{20000 A}{1 A}\right) \cdot \dot{B_{p}} \cdot 2 \pi \cdot \sqrt{a \cdot b}}$ & $(4)$ \\
\hline
\end{tabular}

The currents in the inner control coils were set to rise exponentially during the current quench to a value of 20 $\mathrm{kA}$. The first $3 \mathrm{~ms}$ of the current quench were simulated with a time step width of $0.05 \mathrm{~ms}$. The mesh was generated with adaptive cell length. The cells were set to be tetrahedral with an angle between $50^{\circ}$ and $60^{\circ}$. A nonlinear residual of 0.005 was set as convergence criterion for each time step.

A simplified model of the assembly 1 was placed into the coil model together with the underlying frame and a slice of the vacuum vessel. Toroidal currents were thus suppressed. Measurements show a peak toroidal current of $350 \mathrm{kA}$ in the vessel. This creates an additional magnetic field of $11 \mathrm{mT}$ at the position of the divertor assembly, resulting in additional forces of up to $700 \mathrm{~N}$ on the support structure and up to $500 \mathrm{~N}$ on the tiles, depending on the case. Hence, the following results underestimate the magnitude of the force due to the suppressed toroidal currents. The materials of the tiles and the sockets were changed according to the investigated case. Seven different cases were simulated:

1. Graphite tiles, stainless steel clamps, both sockets isolated (1997-2013)

2. Tungsten tiles, stainless steel clamps, left socket isolated (2015-2018)

3. Tungsten tiles, stainless steel clamps, right socket isolated (2015-2018)

4. Tungsten tiles, stainless steel clamps, both sockets conducting (2014-2015)

5. Tungsten tiles, titanium clamps, left socket isolated

6. Tungsten tiles, titanium clamps, both sockets isolated (new 2018)

7. Tungsten tiles, titanium clamps, both sockets conducting

The first four cases represent the original version of assembly 1 and the modifications to tiles and sockets. Case 2, being the most recent setup, will be used as reference. The last three cases were investigated as options and case 6 was implemented in the 2018 maintenance break.

Fig. 3 shows the time traces of the force in $x$-direction for each component. The traces are similar for all cases and poloidal field variations. This is also true for the torques. However, magnitude and direction of the forces and torques change depending on the change rate of the poloidal magnetic field, the material of the tiles/clamps, as well as which socket is insulated. These 
1 highest loads occurred within the first $0.5 \mathrm{~ms}$ of the

2 current quench for all cases and current quench times.

3 A $10 \mathrm{~ms}$ simulation revealed no further peak loads.

4 Delayed electromagnetic transients of the structures

5 were neglected.

6 Fig. 4 shows the results for the maximum total forces (a)

7 and torques (b) on the assembly, the frame and the tiles

8 for all cases at $\dot{B}_{p}=200 \mathrm{~T} / \mathrm{s}$. It can be seen that case 1

9 has the lowest forces in all components. For the tiles,

10 this is due to the higher electrical resistivity of graphite

11 compared to tungsten. The forces in the assembly and

12 the frame are low compared to case 2 because of the

13 absence of current loops. The low torque on the

14 assembly supports this. In case 2 the forces on the tiles

15 which are located at the sides of the cooling plates $(1$,

$164,5,8)$ are strongly elevated.

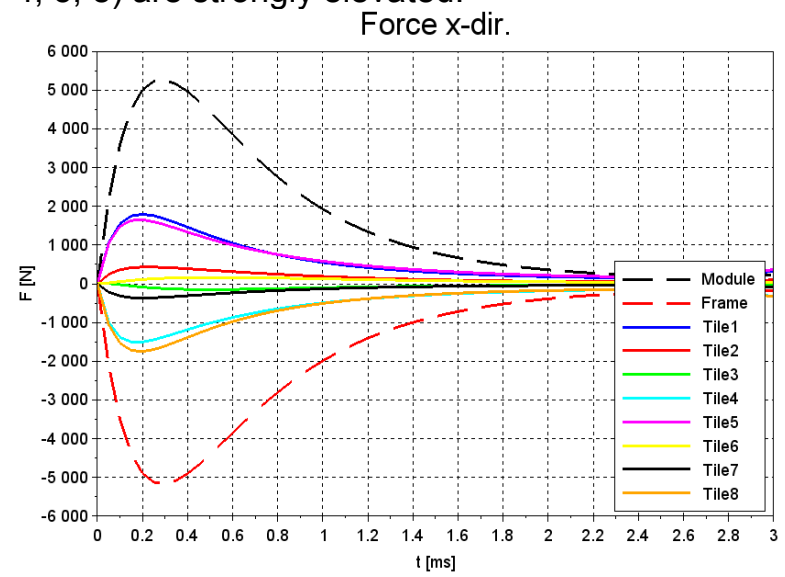

the single components for case 5 at at $\dot{B}_{p}=200 \mathrm{~T} / \mathrm{s}$ This is caused by two current loops, which run from the
cooling plates through the outmost clamps, and tiles back into the cooling plates (Fig. 5a). This parasitic current in the outer tiles gives rise to this strong jxB force. A second current loop, running from the support structure through the right socket, the vacuum vessel and the frame back into the structure (Fig. 5b), is responsible for the strong forces on the assembly and the frame, as well as the higher torque on the assembly. A similar behavior can be seen in case 3 . Forces and torques on the tiles are very similar. The forces on the frame and the assembly are lower than in case 2 because the current loop is now running through the left socket, counteracting independent eddy currents in the components. In case 4 forces and torques on the tiles are again close to the values in case 2 . The forces on the assembly and the frame however are strongly reduced compared to case 2 . Reason for this is that the current loop is now running through the entire length of the support structure, through both sockets and the vacuum vessel. The current is therefore highly symmetrical in the assembly and the frame which results in very low forces. However, the strongest torques occur in this case as the current loops are very large compared to all other cases. In case 5 the forces on the tiles are significantly reduced. This due to the higher electrical resistivity of the titanium clamps which reduce the parasitic current. The influence on the torques on the tiles is not as pronounced. This indicates that these torques are dominated by the eddy currents in the tiles. The forces and torques on the assembly and the frame in this case are on the same level as in case 2 as the same current loop occurs. Case 6 shows the same reduced tile forces due to the titanium clamps as
54 in the previous case. Forces and torques are similar the 55 values in case 1 . This is because both cases have 56 isolated sockets and therefore no current loops. Case 7 57 also proves the effect of the titanium clamps on the tile 58 forces. Concerning forces and torques on the assembly 59 and the frame it can be seen that the same levels are 60 reached as in case 4 . This is again due to the large 61 current loop through both sockets, the entire support 62 structure and the vacuum vessel.

63
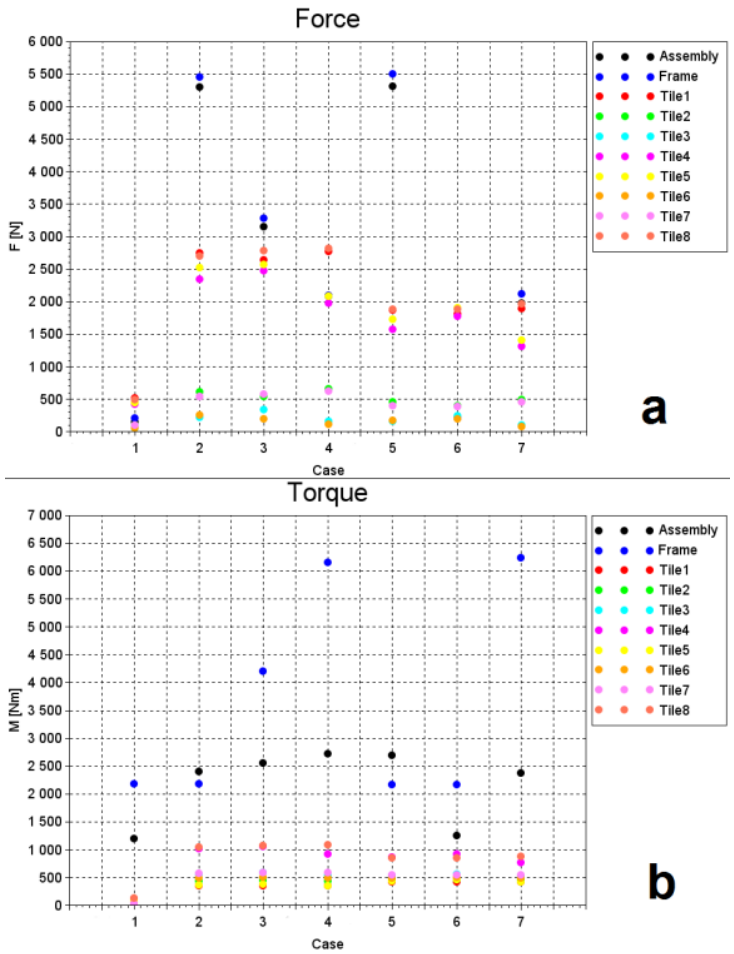

64

Fure 4: Maximal total forces (a) and torques (b) during current quench on assembly, frame and tiles for various cases at $\dot{B}_{p}=200 \mathrm{~T} / \mathrm{s}$
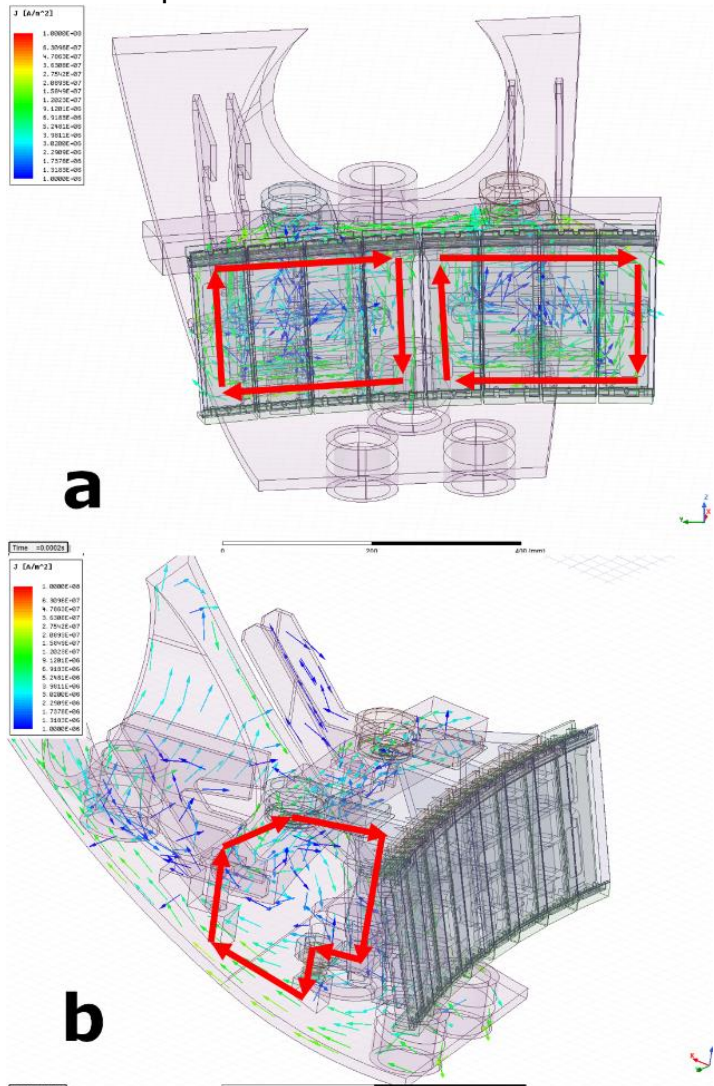

Figure 5: Current loops in the cooling plates/tiles (a) and 70 current loops in the assembly/frame (b) for case 3 at $\dot{B}_{p}=$ $71200 \mathrm{~T} / \mathrm{s}$ 
1 Forces (Fig. 6) and torques become stronger with 2 increasing $\dot{B}_{p}$. The increase of forces and torques 3 becomes smaller at high $\dot{B}_{p}$ though. This asymptotic 4 behavior is due to the B-field, which is generated by the 5 induced current itself. This secondary B-field is 6 established while the poloidal field decays, 7 compensating parts of the poloidal field change.

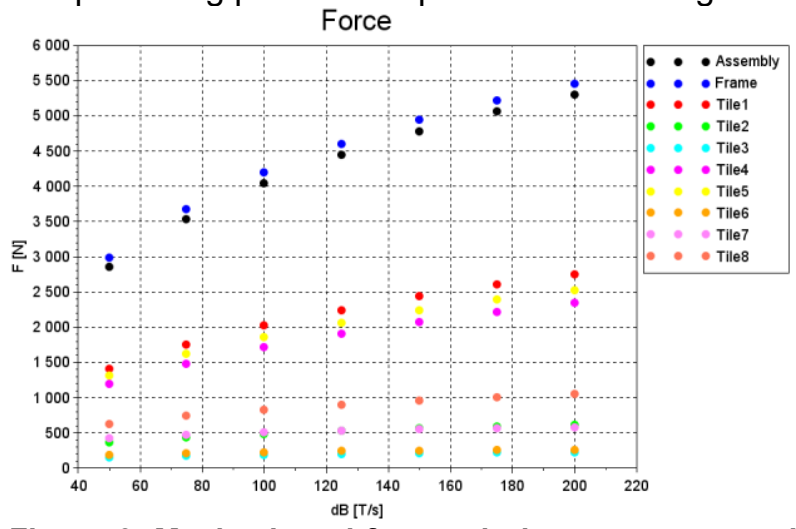

Figure 6: Maximal total forces during current quench on assembly, frame and tiles for case 2 at various $\dot{B}_{p}$

\section{III. FINITE ELEMENT MODEL}

12 This investigation was done in ANSYS transient 13 structural environment. The simplified 3D model of the assembly 1 one was used with the materials according to the cases. The water flanges were set to be fixed while the sockets had a friction boundary condition with the friction coefficients of stainless steel $(0.2)$ or $\mathrm{SiN}$ (0.12) depending on the case. The normal force on the sockets was set to $60 \mathrm{kN}$. The time traces of the forces and torques were imported from the electromagnetic results. The maximal edge length of the tetrahedral mesh was set to be $5 \mathrm{~mm}$, approximating the mesh from electromagnetic analysis. The time step was set to be $0.05 \mathrm{~ms}$ and the total simulation was $3 \mathrm{~ms}$ to agree with the temporal resolution of the EM calculation. A direct solver was used and the convergence criteria were adapted by the solver during calculation.

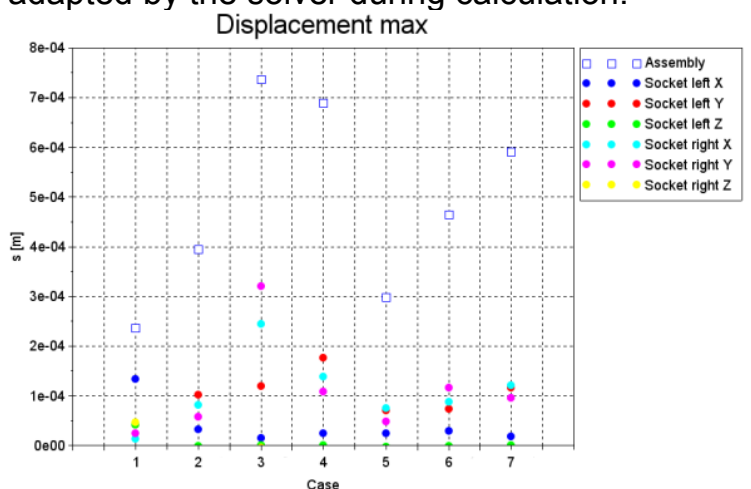

Figure 7: Maximal displacements of the assembly and the sockets for the different cases at $\dot{B}_{p}=200 \mathrm{~T} / \mathrm{s}$

31 Fig. 7 shows the maximal displacements for the entire 32 assembly and the sockets for the different cases and for 33 different $\dot{B}_{p}$ in case 2 . It can be seen that in all cases 34 movement of the sockets in z-direction is widely 35 suppressed by the normal force while displacement in 36 radial $(\mathrm{x})$ and toroidal $(\mathrm{y})$ directions occur regardless of 37 the material pairing at the sockets. This indicates that 38 the friction force at the sockets plays a minor role 39 compared to the forces acting on the assembly. A 40 correlation between the displacement and the forces 41 and torques shown in Fig. 4 cannot be seen. This is 42 because the directions of the forces and torques
43 coincide in cases $3,4,7$, leading to larger 44 displacements, while differing in cases $2,5$.

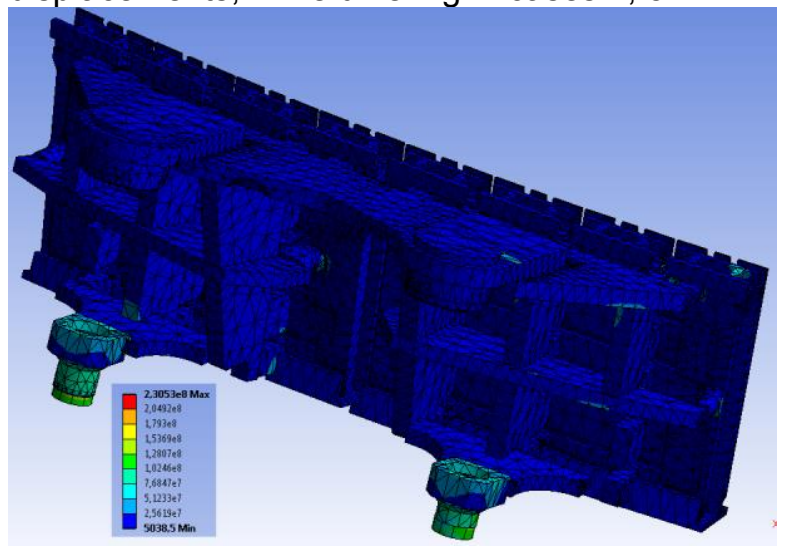

Figure 8: Peak equivalent stress in the assembly during 47 current quench for case 5 and $\dot{B}_{p}=200 \mathrm{~T} / \mathrm{s}$

48 Fig. 8 shows the peak equivalent stress on the 49 assembly for case 2 and a poloidal magnetic field 50 change of $\dot{B}_{p}=200 \mathrm{~T} / \mathrm{s}$. It can be seen that the highest 51 stress occurs in the sockets. With a peak value of 179 $52 \mathrm{MPa}$ and an average stress of $120 \mathrm{MPa}$ it is well below 53 the yield strength of $210 \mathrm{MPa}$ for the material.

54

\section{IV. CONCLUSION AND OUTLOOK}

56 The movement of assembly 1 of the AUG divertor has 57 been investigated using a $3 \mathrm{D}$ transient model for 58 calculating electromagnetic forces and torques. The 59 results pointed out current loops inside the assembly 60 and through the surrounding structures which give rise 61 to forces up to $5.5 \mathrm{kN}$. The friction coefficients between $62 \mathrm{SiN}$ and stainless steel were determined to be $0.18 / 0.16$ 63 for $F_{N}<=5 \mathrm{kN}$ and $0.12 / 0.11$ for $F_{N}>5 \mathrm{kN}$. With the 64 forces and torques from the electromagnetic calculation 65 and the friction coefficients, a finite element calculation 66 was performed. The results show large displacements 67 in cases with current loops through surrounding 68 structures. It was decided to implement case 6 for the 69 experimental campaign 2018 as it promises the largest 70 reduction of forces and torques on the assembly and the 71 frame. Both sockets of the assembly 1 are isolated from 72 the vessel to suppress current loops. Furthermore, the 73 stainless steel clamps are replaced with clamps made 74 of titanium Grade5 to reduce the parasitic current 75 through the tiles.

\section{REFERENCES}

[1]K. Krieger, H. Maier, R. Neu, Conclusions about the use of tungsten in the divertor of ASDEX Upgrade Journ. Of Nucl. Mat. Vol 266-269 (1999), p. 207216, https://doi.org/10.1016/S0022-3115(98)00890-3

[2]M.A. Mahdavi, S.L. Allen, D.R.Baker, B. Bastasz, N.H.Brooks, Divertor heat and particle control experiments onthe DIII-D tokamak, Journ. Of Nucl. Mat. Vol 220-222 (1995), p. 13-24, https://doi.org/10.1016/0022-3115(94)00443-9

[3]R. Neu, K. Asmussen, K. Krieger, A. Thoma, H-S Bosch, The tungsten divertor experiment at ASDEX Upgrade, Plasma Phys. Contr. Fusion 38 (1996), A165-A179, https://doi.org/10.1088/0741-3335/38/12A/013

[4]R. Neu, M. Balden, V. Bobkov, R. Dux, O. Gruber, Plasma wall interaction and its implication in an all tungsten divertor tokamak, Plasma Phys. Contr. Fusion 49 (2007), B59-B70, https://doi.org/10.1088/0741-3335/49/12B/S04

[5]A. Herrmann, H. Greuner, N. Jaksic, M. Balden, A. Kallenbach, Solid tungsten Divertor-III for ASDEX Upgrade and contributions to ITER, Nucl. Fusion 55 (2015), 063015, https://doi.org/10.1088/0029-5515/55/6/063015

92 [6]B. Streibl, P. Lang, F. Leutner, J. Noterdaeme, A. Stäbler, Chapter 2: Machine 93 Design, Fueling, and Heating in ASDEX Upgrade, Fus. Sc. and Tech. 44 (2003), 578-592, https://doi.org/10.13182/FST03-A400

95 [7]V.D. Pustitov, G. Rubinacci,, F. Villone, On the computation of the disruption 96 forces in tokamaks, Nucl. Fusion. $\mathbf{5 7}$ (2017), 126038, https://doi.org/10.1088/1741-4326/aa8876 